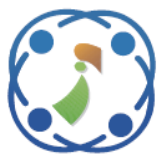

\title{
Darts Game Optimizer: A New Optimization Technique Based on Darts Game
}

\author{
Mohammad Dehghani ${ }^{1 *} \quad$ Zeinab Montazeri $^{1} \quad$ Hadi Givi $^{2}$ \\ Josep M. Guerrero ${ }^{3} \quad$ Gaurav Dhiman ${ }^{4}$ \\ ${ }^{I}$ Department of Electrical and Electronics Engineering, Shiraz University of Technology, Shiraz, Iran \\ ${ }^{2}$ Department of Electrical Engineering, Faculty of Engineering, \\ University of Shahreza, Shahreza 86481-41143, Iran \\ ${ }^{3}$ Center for Research on Microgrids (CROM), Department of Energy Technology, \\ Aalborg University, Aalborg, Denmark \\ ${ }^{4}$ Department of Computer Science, Government Bikram College of Commerce, Patiala, Punjab 147004, India \\ * Corresponding author's Email: adanbax@gmail.com
}

\begin{abstract}
In this paper, a novel game-based optimization technique entitled darts game optimizer (DGO) is proposed. The novelty of this investigation is DGO designing based on simulating the rules of Darts game. The key idea in DGO is to get the most possible points by the players in their throws towards the game board. Simplicity of equations and lack of control parameters are the main features of the proposed algorithm. The ability and quality of DGO performance in optimization is evaluated on twenty-three objective functions, and then is compared with eight other optimization algorithms including Genetic Algorithm (GA), Particle Swarm Optimization (PSO), Gravitational Search Algorithm (GSA), Teaching Learning-Based Optimization (TLBO), Grey Wolf Optimizer (GWO), Grasshopper Optimization Algorithm (GOA), Whale Optimization Algorithm (WOA), and Marine Predators Algorithm (MPA). The results of simulation and comparison indicate the superiority and optimal quality of the proposed DGO algorithm over the mentioned algorithms.
\end{abstract}

Keywords: Optimization, Optimizer, Darts game, Darts game optimizer, Game-based algorithm.

\section{Introduction}

\subsection{Motivation}

There are many optimization problems in different disciplines of science and technology that need to be solved using appropriate optimization methods. Hence, employing an effective optimization algorithm is of great importance for solving such problems. In this regard, optimization algorithms have been applied by scientists in various fields such as energy [1], protection [2], electrical engineering [3-6], energy carriers [7,8], and data mining [9] to achieve the optimal solution. This issue motivates researchers to focus on optimization studies, modification of existing methods, and especially introduction of new optimization methods.

\subsection{Background}

In general, optimization algorithms can be categorized into four groups including physics-based, swarm-based, evolutionary-based, and game-based algorithms.

Physics-based algorithms are designed based on simulation and application of existing laws in physics. For example, the spring search algorithm (SSA) is designed using Hawk's law in the weight and spring system. In SSA, the members of the population are a number of weights that are connected to each other by a spring and the optimal answer is provided by reaching the equilibrium point $[10,11]$. Some of the other algorithms in this category are Ray Optimization (RO) algorithm [12], Black Hole (BH) algorithm [13], Artificial Chemical Reaction Optimization Algorithm (ACROA) [14], Charged 
System Search (CSS) [15], Curved Space Optimization (CSO) [16], Galaxy-based Search Algorithm (GbSA) [17], Small World Optimization Algorithm (SWOA) [18], and Gravitation Search Algorithm (GSA) [19].

Swarm-based algorithms have been introduced based on careful attention towards natural phenomena such as animal social behaviors, insects' behaviors, and various plant processes. Particle swarm optimization (PSO) is one of the most popular techniques in this category. Simulation of movements and behaviors of birds has been used in PSO designing [20]. Artificial Bee Colony (ABC) [21], Spotted Hyena Optimizer (SHO) [22], Bat-inspired Algorithm (BA) [23], Emperor Penguin Optimizer (EPO) [24], Cuckoo Search (CS) algorithm [25], 'Following' Optimization Algorithm (FOA) [26], Grey Wolf Optimizer (GWO) [27], Group Optimization (GO) [28], Donkey Theorem Optimization (DTO) [29], Rat Swarm Optimizer (RSO) [30], Grasshopper Optimization Algorithm (GOA) [31], and Ant Colony Optimization (ACO) $[32,33]$ also belong to this category.

In Evolutionary-based algorithms, evolution of a population is considered in order to create new generations of genetically superior individuals [34]. Genetic Algorithm (GA) [35], which is one of the oldest-known techniques is among these algorithms. The GA is designed by simulating three phases of selection, crossover, and mutation. Some other algorithms of this type are Evolution Strategy (ES) [36], Biogeography-based Optimizer (BBO) [37], Genetic Programming (GP) [38], and Differential Evolution (DE) [39].

Game-based algorithms have been proposed based on modeling the rules and the behavior of players in different games. For example, Shell Game Optimization (SGO) [40] is based on shell game modeling. In SGO, players try to find the object hidden under one of the three shells. Orientation Search Algorithm (OSA) [41, 42], Hide Objects Game Optimization (HOGO) [43], and Dice Game Optimizer (DGO) [44] are also in this category.

\subsection{Contribution}

Numerous studies and investigations have been conducted by researchers in the field of optimization to present effective optimization algorithms. Although different games can be a good source for designing optimization algorithms, researchers have paid less attention to this potential. Therefore, the authors of this article are interested in designing a game-based algorithm.
This study proposes a new game-based optimization algorithm called Darts Game Optimizer (DGO), which is designed based on simulation of the attractive Darts game. The population members in the proposed algorithm are darts players, who try to collect the most points in their throws towards the game board. In optimization problems, this effort can be modelled to obtain an algorithm to reach the optimal solution.

\subsection{Paper structure}

In the continuation of this article, darts game is first introduced in section 2 . The design steps of the proposed algorithm are described in Section 3. The simulation and evaluation of the proposed algorithm are accomplished in Section 4. Finally, conclusions and suggestions are expressed in Section 5.

\section{Darts game}

Darts is a sport that everyone enjoys it regardless of age or gender. Although this game seems simple, the skill of the players has an important effect on the collection of points. The equipment of Darts game includes a dartboard and darts, which are shown in Fig. 1. Based on the division, the dartboard has 82 areas with different points. According to Fig. 2, the scoring method for each throw is that the inner bull has 50 and the outer bull has 25 points. The score of each sector is written above it. If the darts hit the inner narrow ring, the score is tripled, and if they hit the outer narrow ring, the score is doubled. The important thing is that the center of the dartboard is the fifth highest scoring area of the game. This issue is explained by the fact that the areas of sectors 20,19 , 18 , and 17 each have 60, 57, 57, and 51 points, respectively.

\section{Darts game optimizer (DGO)}

In this section, the potential of Darts game is

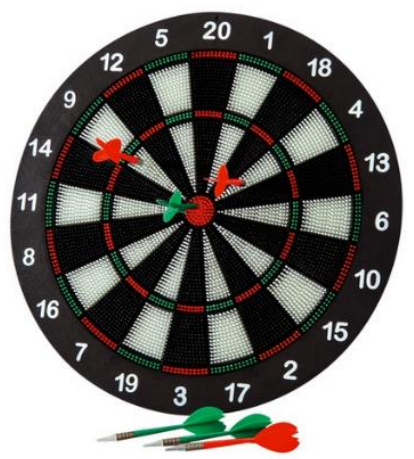

Figure. 1 Equipment of darts game 


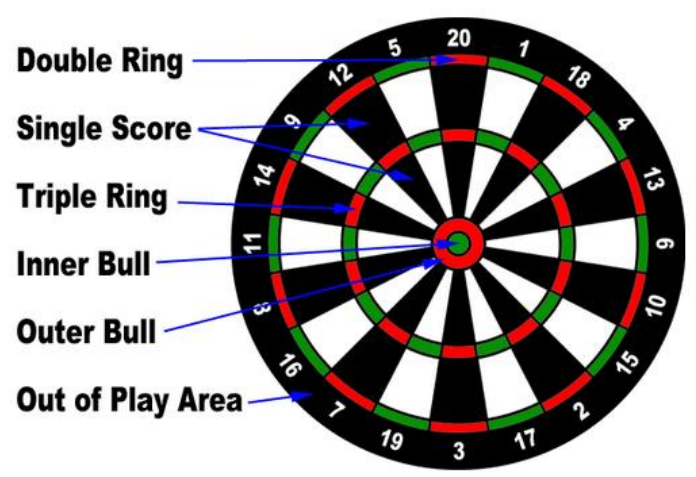

Figure. 2 Scoring method for darts game

applied to design and introduce a new optimizer. Searcher agents in DGO are the players in this game and their goal is to get the highest score (optimal answer).

\subsection{Mathematical modelling}

The population of the players is modeled with a matrix, each row of which represents one player and each column represents the different characteristics of each player. The number of columns in this matrix is actually the same as the number of problem variables and the values suggested for these variables. The players' matrix is specified using (1).

$$
X=\left[\begin{array}{c|ccccc}
X_{1} & x_{1}^{1} & \cdots & x_{1}^{d} & \cdots & x_{1}^{m} \\
\vdots & \vdots & \ddots & \vdots & \ddots & \vdots \\
X_{i} & x_{i}^{1} & \cdots & x_{i}^{d} & \cdots & x_{i}^{m} \\
\vdots & \vdots & \ddots & \vdots & \ddots & \vdots \\
X_{N} & x_{N}^{1} & \cdots & x_{N}^{d} & \cdots & x_{N}^{m}
\end{array}\right]
$$

Here $X$ is the players' matrix, $x_{i}^{d}$ is the $d$ th dimension of $i$ th player, $m$ is the number of variables, and $N$ is the number of players.

By placing $X_{i}$ in the fitness function, useful information is obtained, which is presented in (2) to (7).

$$
\begin{aligned}
& F_{\text {best }}=\min (f i t)_{N \times 1} \\
& X_{\text {best }}=X(\text { location of } \min (f i t), 1: m) \\
& F_{\text {worst }}=\max (f i t)_{N \times 1} \\
& X_{\text {worst }}=X(\text { location of } \max (f i t), 1: m) \\
& F^{n}=\frac{f i t-F_{\text {worst }}}{\sum_{j=1}^{N}\left(\text { fit }_{j}-F_{\text {worst }}\right)} \\
& P_{i}=\frac{F_{i}^{n}}{\max \left(F^{n}\right)}
\end{aligned}
$$

Table 1. Information of different sectors in the dartboard

\begin{tabular}{|c|c|c|}
\hline sector & $\begin{array}{c}\text { Angel interval } \\
\text { (degree) }\end{array}$ & Area $\left(\mathrm{mm}^{2}\right)$ \\
\hline Inner bull & $0 \leq \theta \leq 360$ & 506.7075 \\
\hline Outer bull & $0 \leq \theta \leq 360$ & $2.6702 \times 10^{+3}$ \\
\hline $\begin{array}{c}\text { Inner single } \\
\text { score }\end{array}$ & $0 \leq \theta \leq 18$ & 910.1131 \\
\hline $\begin{array}{c}\text { Outer single } \\
\text { score }\end{array}$ & $0 \leq \theta \leq 18$ & $5.3535 \times 10^{+3}$ \\
\hline double ring & $0 \leq \theta \leq 18$ & 556.6902 \\
\hline treble ring & $0 \leq \theta \leq 18$ & 278.9734 \\
\hline
\end{tabular}

Here $F_{\text {best }}$ represents the best fitness function value, $X_{\text {best }}$ is the best variables' values, $F_{\text {worst }}$ is the worst fitness function value, $X_{\text {worst }}$ is the worst variables' values, $F^{n}$ is the normalize value of fitness functions, and $P_{i}$ is the probability function of $i$ th player.

For all dartboards, dimensions follow a standard as follows:

- Inside measurement of double and treble ring: $8 \mathrm{~mm}$

- Inside diameter of bull: $12.7 \mathrm{~mm}$

- Inside diameter of outer bull: $31.8 \mathrm{~mm}$

- Center bull to the inside edge of treble wire: $107 \mathrm{~mm}$

- Center bull to outside edge bull wire: $170 \mathrm{~mm}$

- Outside edge of the double wire to outside edge of double wire: $340 \mathrm{~mm}$

- Overall dartboard diameter: $451 \mathrm{~mm}$

As mentioned in the previous section, the Dartboard has 82 areas with different scores. Each player can throw three darts in each iteration. The location of the darts on the Dartboard depends on two factors: player skill and chance.

There are six types of sectors with different areas on the Dartboard as specified in Table 1.

Therefore, the throwing score is modelled and calculated for each player using (8) to (11).

$$
\begin{gathered}
C_{i}=\operatorname{round}\left(82 \times\left(1-P_{i}\right)\right) \\
S C_{i}=\left\{\begin{array}{c}
S(1: C), \text { rand }<P_{i} \\
S(C+1: 82), \text { else }
\end{array}\right. \\
S_{i}=S C_{i}(k) \& 1 \leq k \leq 82 \\
s_{i}^{n}=\frac{\sum_{\text {throws }=1}^{3} s_{i}^{\text {throws }}}{180}
\end{gathered}
$$

Here $S C_{i}$ denotes the score candidates for $i$ th player, $S$ is the score matrix, which is sorted from high scores to low scores, $s_{i}$ is the score for each throw of $i$ th player, and $s_{i}^{n}$ is the normalized score of $i$ th player. 
Finally, the new status of each player and in fact, the values of the problem variables are updated using (12).

$$
X_{i}=X_{i}+\operatorname{rand}(1, m) \times\left(X_{\text {best }}-3 s_{i}^{n} X_{i}\right)
$$

\subsection{Steps of DGO}

After modelling the proposed DGO algorithm, it can be used to solve various optimization problems.

The DGO has some basic parameters that need to be determined. The number of members in the algorithm population is 50 players and the number of repetitions of the algorithm as a stop condition for the algorithm is considered as 1000 repetitions. The initial population of players is created at random at the beginning of the algorithm.

Each player in the suggested algorithm is actually an m-member vector that represents a suggested answer to the problem. The members of this vector show the problem variables that are evaluated by placing them in the objective function.

The various steps in implementing the DGO are as follows:

Start DGO

Step1: Creating the initial population of players.

Step2: Calculating the fitness function.

Step3: Updating $F_{\text {best }}, X_{\text {best }}, F_{\text {worst }}$, and $X_{\text {worst }}$ using (2) to (5).

Step4: Updating $F^{n}$ and $P_{i}$ using (6) and (7).

Step5: Calculating $s_{i}^{n}$ using (8) to (11).

Step6: Updating $X_{i}$ using (12).

Step7: Checking the stop condition.

Step8: Printing solution.

End DGO

\section{Simulation study and discussion}

In this section, the performance of DGO is evaluated and compared with eight other algorithms considering twenty-three standard objective functions. These objective functions are categorized into Unimodal [45,46], Multimodal [46,47], and Fixed-dimension Multimodal [46]. Eight algorithms including GA, PSO, GSA, TLBO, GWO, GOA, WOA, and MPA are considered to compare the optimization results. The average (Ave) and standard deviation (std) of the best optimal solution are utilized for evaluation.

\subsection{Performance evaluation on unimodal functions F1-F7}

The objective functions F1 to F7 are used to evaluate the exploitation ability of optimization algorithms in achieving the optimal response. Table
2 presents the results of testing the proposed algorithm and the eight aforementioned algorithms on these functions, which indicates the significant superiority of the proposed algorithm for this type of objective functions.

\subsection{Performance evaluation on multimodal functions $\mathrm{F}_{8}-\mathrm{F}_{13}$}

Objective functions F8 to F13 are in this group, which have several local solutions. Therefore, by implementing optimization algorithms on this type of objective functions, exploration capability can be evaluated. The results of this evaluation are presented in Table 3, which shows the strong performance of the proposed algorithm compared to the other algorithms.

\subsection{Performance evaluation on fixed-dimension multimodal functions $F_{14}-F_{23}$}

DGO and eight other algorithms have been evaluated on the functions in this category. These functions have lower dimension of variables in comparison with the previous two categories. The obtained results in Table 4 indicate the superiority of the proposed algorithm for F14 to F23.

\subsection{Theoretical analysis}

As can be seen from the numerical analysis, the proposed DGO algorithm has an acceptable advantage over the other eight algorithms. The two most important indicators for evaluating exploration algorithms are exploitation and exploration capacities.

Exploitation capacity indicates the algorithm's ability to achieve the optimal answer. The Unimodal objective functions are used to evaluate this index. The results in Table 2 indicate the appropriate exploitation capacity of the proposed DGO algorithm compared to other algorithms.

Exploratory capacity indicates the accurate search of the search space, which prevents the algorithm from getting stuck in a local area. The Multimodal and Fixed-dimension Multimodal test functions are used to evaluate this index. The results in Table 3 and Table 4 indicate the appropriate exploration capacity of the proposed algorithm compared to other algorithms.

The authors have been working in the field of optimization to develop game-based optimization algorithms. Several algorithms such as OSA, BOSA, HOGO, and SGO have been proposed in our previous researches. In the study of optimization algorithms, it is important to note that no algorithm is necessarily the best one. Optimization algorithms provide quasi- 
optimal answers, so researchers try to propose new algorithms to improve the efficiency. The main advantage of the proposed algorithm, in addition to its superiority in numerical analysis, is the simplicity of the equations and its implementation, as well as the lack of any control parameters.

\section{Conclusions}

The authors' contribution in this paper is presenting a new game-based optimization algorithm named Darts Game Optimizer (DGO). The innovation of the proposed method is design of a new optimization technique based on simulating the Darts game, in which players try to get the most points from Dart's throws. The mathematical modelling and the steps of implementing the proposed algorithm were thoroughly described. In order to evaluate the proposed algorithm, a collection of twenty-three objective functions were considered as case studies in three different categories including unimodal, Multimodal, and Fixed-dimension Multimodal test functions. DGO was implemented on this set of objective functions, and to show its effectiveness, the results were compared with eight other algorithms including Genetic Algorithm (GA), Particle Swarm Optimization (PSO), Gravitational Search Algorithm (GSA), Teaching Learning Based Optimization (TLBO), Grey Wolf Optimizer (GWO), Grasshopper Optimization Algorithm (GOA), Whale Optimization Algorithm (WOA), and Marine Predators Algorithm (MPA). The results confirmed the superiority of the proposed algorithm and its ability of exploration and exploitation for solving different optimization problems compared to the mentioned algorithms.

In addition to the results of experiments and numerical analysis that indicated the superiority of the DGO algorithm, a theoretical analysis was performed on the proposed algorithm, which represented excellence and features of the DGO algorithm such as simplicity of the equations and implementation, lack of any control parameters, and good exploitation and exploration capacities.

For future works, the authors propose several ideas for investigation. One can create a binary variant of DGO as an important potential contribution. DGO may also be used to overcome many-objective real-life optimization as well as multi-objective problems.

\section{Conflicts of Interest}

The authors declare no conflict of interest.

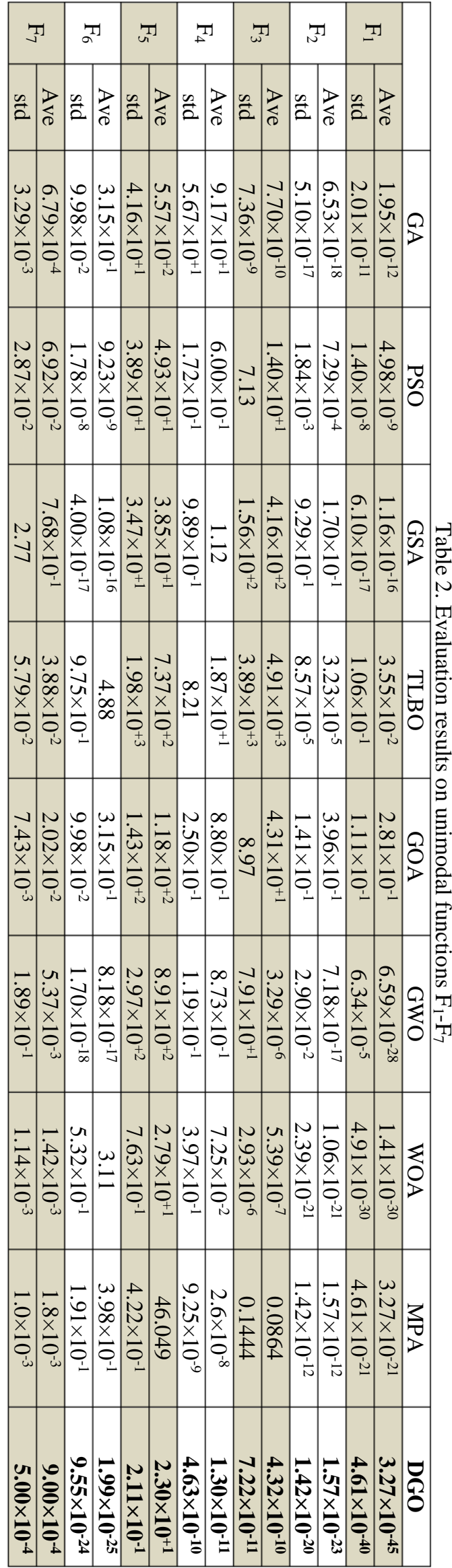




\begin{tabular}{|c|c|c|c|c|c|}
\hline$\underset{\omega}{T}$ & $\frac{T}{N}$ & $=$ & $\frac{T}{6}$ & ${ }_{0}^{T}$ & ${ }_{\infty}^{T}$ \\
\hline & & & & & $\frac{1}{2}$ \\
\hline
\end{tabular}

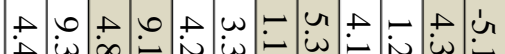

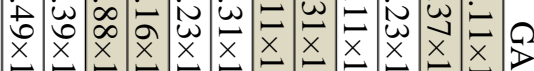

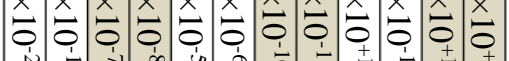

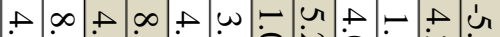

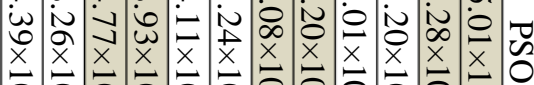

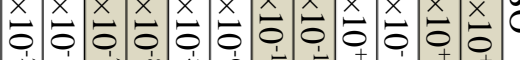

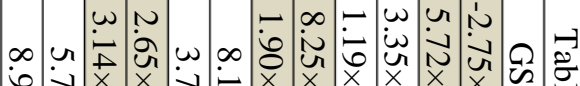

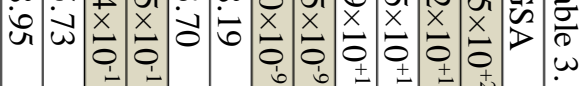

$\infty N$ N n

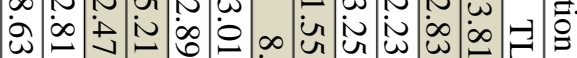

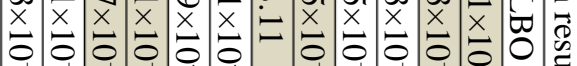

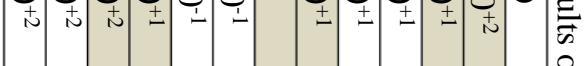

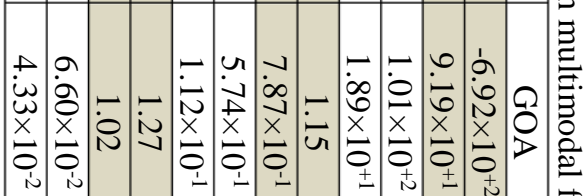

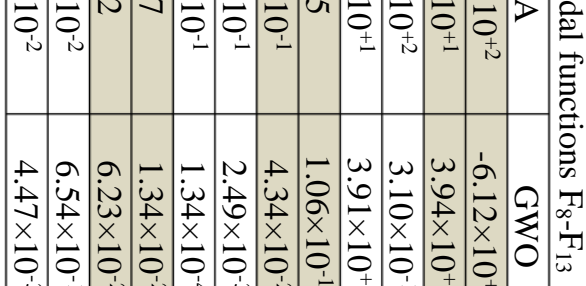

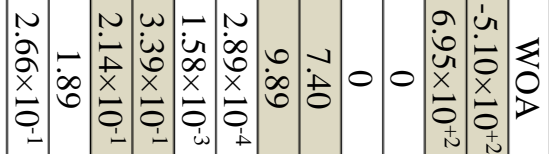

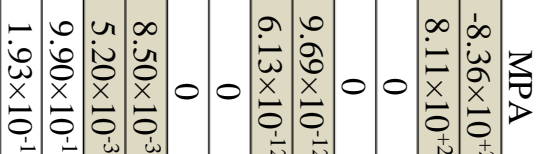

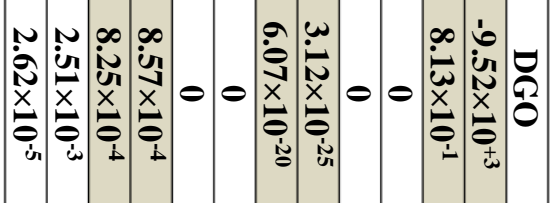

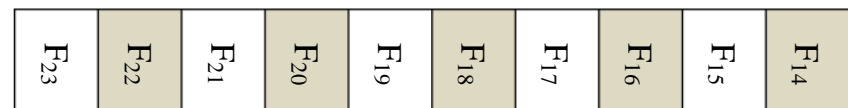

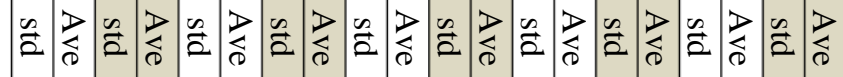

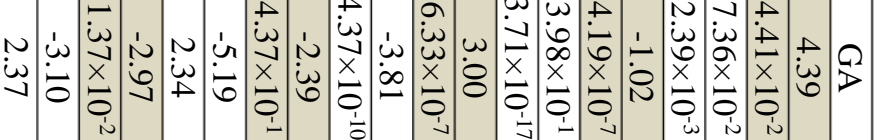

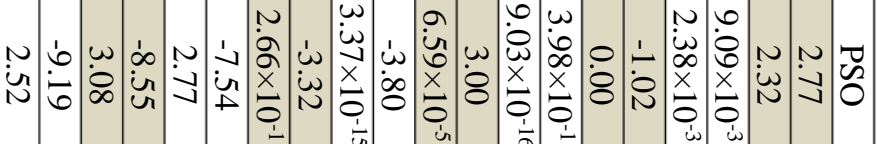

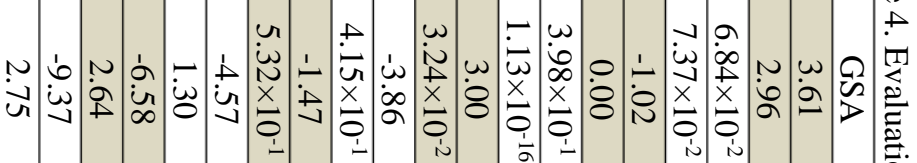

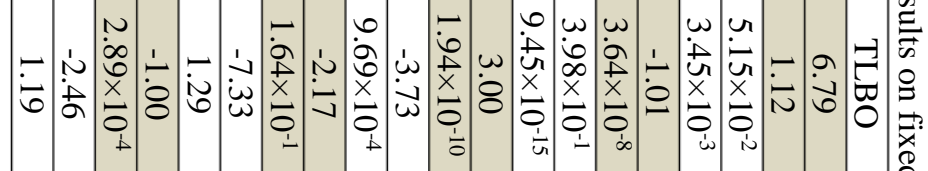

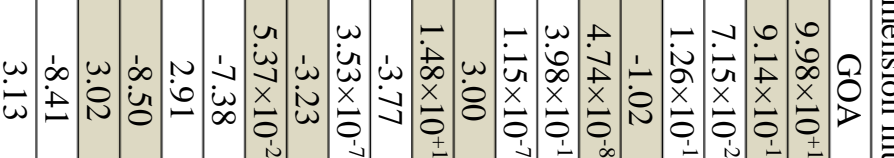

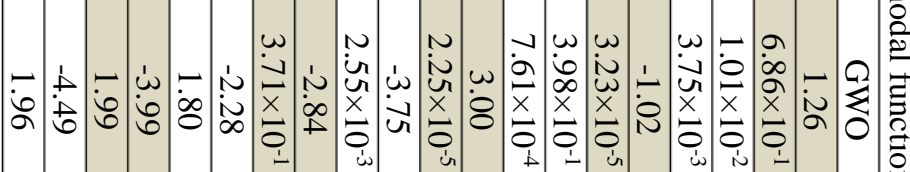

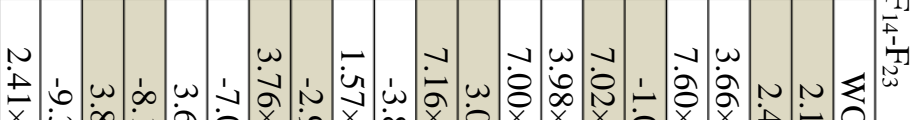

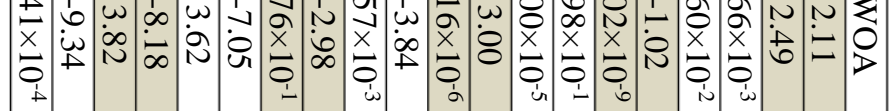

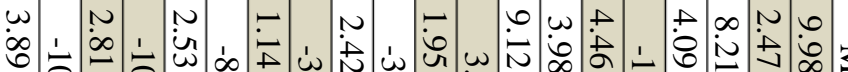

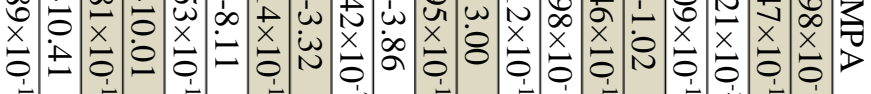

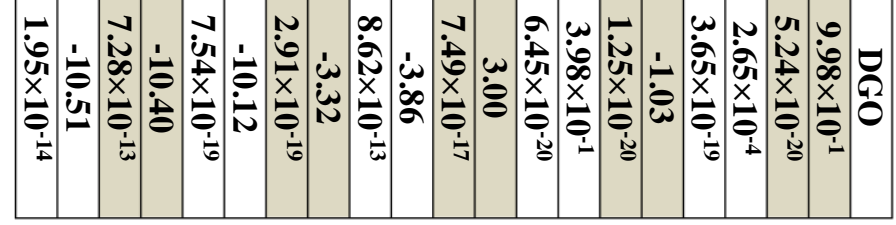




\section{Author Contributions}

Conceptualization, M. Dehghani, Z. Montazeri, and J. M. Guerrero.; methodology, M. Dehghani and Z. Montazeri.; software, M. Dehghani and H. Givi.; validation, J. M. Guerrero, H. Givi, and G. Dhiman.; formal analysis, H. Givi and G. Dhiman.; investigation, M. Dehgani and Z. Montazeri.; resources, J. M. Guerrero.; data curation, H. Givi and G. Dhiman; writing - original draft preparation, $\mathrm{M}$. Dehghani and Z. Montazeri.; writing-review and editing, H. Givi and G. Dhiman.; visualization, M. Dehghani.; supervision, M. Dehghani.; project administration, M. Dehghani and Z. Montazeri; funding acquisition, J. M. Guerrero.

\section{Acknowledgments}

J. M. Guerrero was supported by VILLUM FONDEN under the VILLUM Investigator Grant (no. 25920): Center for Research on Microgrids (CROM); www.crom.et.aau.dk

\section{References}

[1] M. Dehghani, Z. Montazeri, and O. P. Malik, "Energy Commitment: A Planning of Energy Carrier Based on Energy Consumption", Электротехника и Электромеханика, №. 4, pp. 69-72, 2019.

[2] A. Ehsanifar, M. Dehghani, and M. Allahbakhshi, "Calculating The Leakage Inductance for Transformer Inter-Turn Fault Detection Using Finite Element Method", In: Proc. of Iranian Conf. on Electrical Engineering (ICEE), Tehran, Iran, pp. 1372-1377, 2017.

[3] M. Dehghani, Z. Montazeri, and O. P. Malik, "Optimal Sizing and Placement of Capacitor Banks and Distributed Generation in Distribution Systems Using Spring Search Algorithm", International Journal of Emerging Electric Power Systems, Vol. 21, No. 1, 20190217, 2020.

[4] M. Dehghani, Z. Montazeri, O. P. Malik, K. AlHaddad, J. M. Guerrero, and G. Dhiman, "A New Methodology Called Dice Game Optimizer for Capacitor Placement in Distribution Systems", Электротехника u Электромеханика, No. 1, pp. 61-64, 2020.

[5] S. Dehbozorgi, A. Ehsanifar, Z. Montazeri, M. Dehghani, and A. Seifi, "Line Loss Reduction and Voltage Profile Improvement in Radial Distribution Networks Using Battery Energy Storage System", In: Proc. of IEEE 4th International Conf. on Knowledge-Based
Engineering and Innovation (KBEI), Tehran, Iran, pp. 0215-0219, 2017.

[6] Z. Montazeri and T. Niknam, "Optimal Utilization of Electrical Energy from Power Plants Based on Final Energy Consumption Using Gravitational Search Algorithm", Электротехника и Электромеханика, No. 4, pp. 70-73, 2018.

[7] M. Dehghani, Z. Montazeri, A. Ehsanifar, A. Seifi, M. Ebadi, and O. Grechko, "Planning of Energy Carriers Based on Final Energy Consumption Using Dynamic Programming and Particle Swarm Optimization", Электротехника и Электромеханика, No. 5, pp. 62-71, 2018.

[8] Z. Montazeri and T. Niknam, "Energy Carriers Management Based on Energy Consumption", In: Proc. of IEEE 4th International Conf. on Knowledge-Based Engineering and Innovation (KBEI), Tehran, Iran, pp. 539-543, 2017.

[9] Y. Djenouri, A. Belhadi, and R. Belkebir, "Bees Swarm Optimization Guided by Data Mining Techniques for Document Information Retrieval", Expert Systems with Applications, Vol. 94, pp. 126-136, 2018.

[10] M. Dehghani, Z. Montazeri, A. Dehghani, N. Nouri, and A. Seifi, "BSSA: Binary Spring Search Algorithm", In: Proc. of IEEE 4th International Conf. on Knowledge-Based Engineering and Innovation (KBEI), Tehran, Iran, pp. 0220-0224, 2017.

[11] M. Dehghani, Z. Montazeri, A. Dehghani, and A. Seifi, "Spring Search Algorithm: A New MetaHeuristic Optimization Algorithm Inspired by Hooke's Law", In: Proc. of IEEE 4th International Conf. on Knowledge-Based Engineering and Innovation (KBEI), Tehran, Iran, pp. 0210-0214, 2017.

[12] A. Kaveh and M. Khayatazad, "A New MetaHeuristic Method: Ray Optimization", Computers \& Structures, Vol. 112, pp. 283-294, 2012.

[13] A. Hatamlou, "Black Hole: A New Heuristic Optimization Approach for Data Clustering", Information Sciences, Vol. 222, pp. 175-184, 2013.

[14] B. Alatas, "ACROA: Artificial Chemical Reaction Optimization Algorithm for Global Optimization", Expert Systems with Applications, Vol. 38, pp. 13170-13180, 2011.

[15] A. Kaveh and S. Talatahari, "A Novel Heuristic Optimization Method: Charged System Search", Acta Mechanica, Vol. 213, pp. 267-289, 2010.

[16] F. F. Moghaddam, R. F. Moghaddam, and M. Cheriet, "Curved Space Optimization: A 
Random Search Based on General Relativity Theory", arXiv preprint arXiv:1208.2214, 2012.

[17] H. Shah-Hosseini, "Principal Components Analysis by the Galaxy-Based Search Algorithm: A Novel Metaheuristic for Continuous Optimisation", International Journal of Computational Science and Engineering, Vol. 6, pp. 132-140, 2011.

[18] H. Du, X. Wu, and J. Zhuang, "Small-World Optimization Algorithm for Function Optimization", In: Proc. of International Conf. on Natural Computation, pp. 264-273, 2006.

[19] E. Rashedi, H. Nezamabadi-Pour, and S. Saryazdi, "GSA: A Gravitational Search Algorithm", Information Sciences, Vol. 179, pp. 2232-2248, 2009.

[20] R. Eberhart and J. Kennedy, "A New Optimizer Using Particle Swarm Theory”, In: Proc. of Sixth International Symposium on Micro Machine and Human Science, pp. 39-43, 1995.

[21] D. Karaboga and B. Basturk, "Artificial Bee Colony (ABC) Optimization Algorithm for Solving Constrained Optimization Problems", In: Proc. of International Fuzzy Systems Association World Congress, pp. 789-798, 2007.

[22] G. Dhiman and V. Kumar, "Spotted Hyena Optimizer: A Novel Bio-Inspired Based Metaheuristic Technique for Engineering Applications", Advances in Engineering Software, Vol. 114, pp. 48-70, 2017.

[23] X.-S. Yang, "A New Metaheuristic Bat-Inspired Algorithm", In: Nature Inspired Cooperative Strategies for Optimization (NICSO 2010), ed: Springer, pp. 65-74, 2010.

[24] G. Dhiman and V. Kumar, "Emperor Penguin Optimizer: A Bio-Inspired Algorithm for Engineering Problems", Knowledge-Based Systems, Vol. 159, pp. 20-50, 2018.

[25] A. H. Gandomi, X.-S. Yang, and A. H. Alavi, "Cuckoo Search Algorithm: A Metaheuristic Approach to Solve Structural Optimization Problems", Engineering with Computers, Vol. 29, pp. 17-35, 2013.

[26] M. Dehghani, M. Mardaneh, and O. P. Malik, "FOA: Following' Optimization Algorithm for Solving Power Engineering Optimization Problems", Journal of Operation and Automation in Power Engineering, Vol. 8, No. 1, pp. 57-64, 2020.

[27] S. Mirjalili, S. M. Mirjalili, and A. Lewis, "Grey Wolf Optimizer", Advances in Engineering Software, Vol. 69, pp. 46-61, 2014.

[28] M. Dehghani, Z. Montazeri, A. Dehghani, and O. P. Malik, "GO: Group Optimization", Gazi University Journal of Science, Vol. 33, 2020.
[29] M. Dehghani, M. Mardaneh, O. P. Malik, and S. M. NouraeiPour, "DTO: Donkey Theorem Optimization", In: Proc. of 27th Iranian Conf. on Electrical Engineering (ICEE), Yazd, Iran, pp. 1855-1859, 2019.

[30] G. Dhiman, M. Garg, A. Nagar, V. Kumar, and M. Dehghani, "A Novel Algorithm for Global Optimization: Rat Swarm Optimizer", Journal of Ambient Intelligence and Humanized Computing, 2020.

[31] S. Saremi, S. Mirjalili, and A. Lewis, "Grasshopper Optimisation Algorithm: Theory and Application", Advances in Engineering Software, Vol. 105, pp. 30-47, 2017.

[32] M. Dorigo and T. Stützle, "Ant Colony Optimization: Overview and Recent Advances", In: Handbook of Metaheuristics, ed: Springer, pp. 311-351, 2019.

[33] H. Givi, M. A. Noroozi, B. Vahidi, J. S. Moghani, and M. A. V. Zand, "A Novel Approach for Optimization of Z-Matrix Building Process Using Ant Colony Algorithm”, Journal of Basic and Applied Scientific Research, Vol. 2, No. 9, pp. 8932-8937, 2012.

[34] N. E. Karkalos, A. P. Markopoulos, and J. P. Davim, "Evolutionary-Based Methods", In: Computational Methods for Application in Industry 4.0, ed: Springer, pp. 11-31, 2019.

[35] K. S. Tang, K.-F. Man, S. Kwong, and Q. He, "Genetic Algorithms and Their Applications", IEEE Signal Processing Magazine, Vol. 13, pp. 22-37, 1996.

[36] H. G. Beyer and H.-P. Schwefel, "Evolution Strategies-A Comprehensive Introduction", Natural Computing, Vol. 1, pp. 3-52, 2002.

[37] S. Mirjalili, "Biogeography-Based Optimisation", In: Evolutionary Algorithms and Neural Networks, ed: Springer, pp. 57-72, 2019.

[38] J. R. Koza, "Genetic Programming: A Paradigm for Genetically Breeding Populations of Computer Programs to Solve Problems", Stanford University, Department of Computer Science, 1990.

[39] R. Storn and K. Price, "Differential Evolution-A Simple and Efficient Adaptive Scheme for Global Optimization over Continuous Spaces [r]", Berkeley: ICSI, 1995.

[40] M. Dehghani, Z. Montazeri, and O. P. Malik, H. Givi, and J. M. Guerrero, "Shell Game Optimization: A Novel Game-Based Algorithm", International Journal of Intelligent Engineering and Systems, Vol. 13, No. 3, pp. 246-255, 2020.

[41] M. Dehghani, Z. Montazeri, O. P. Malik, A. Ehsanifar, and A. Dehghani, "OSA: Orientation Search Algorithm", International Journal of 
Industrial Electronics, Control and Optimization, Vol. 2, No. 2, pp.99-112, 2019.

[42] M. Dehghani, Z. Montazeri, O. P. Malik, G. Dhiman, and V. Kumar, "BOSA: Binary Orientation Search Algorithm", International Journal of Innovative Technology and Exploring Engineering (IJITEE), Vol. 9, pp. 5306-5310, 2019.

[43] M. Dehghani, Z. Montazeri, S. Saremi, A. Dehghani, O. P. Malik, K. Al-Haddad, J. M. Guerrero, "HOGO: Hide Objects Game Optimization", International Journal of Intelligent Engineering and Systems, Vol. 13, 2020.

[44] M. Dehghani, Z. Montazeri, and O. P. MALIK, "DGO: Dice Game Optimizer", Gazi University Journal of Science, Vol. 32, pp. 871-882, 2019.

[45] J. G. Digalakis and K. G. Margaritis, "On Benchmarking Functions for Genetic Algorithms", International Journal of Computer Mathematics, Vol. 77, No. 4, pp. 481-506, 2001.

[46] G. G. Wang, A. H. Gandomi, X.-S. Yang, and A. H. Alavi, "A Novel Improved Accelerated Particle Swarm Optimization Algorithm for Global Numerical Optimization", Engineering Computations, Vol. 31, No. 7, pp. 1198-1220, 2014.

[47] X. S. Yang, "Firefly Algorithm, Stochastic Test Functions and Design Optimisation", International Journal of Bio-Inspired Computation, Vol. 2, No. 2, pp. 78-84, 2010. 